\title{
ASYMPTOTIC PATHS FOR SUBHARMONIC FUNCTIONS IN $R^{n}$
}

\author{
LENNART CARLESON
}

1. The purpose of this note is to prove the following theorem.

Theorem. Let $u(x)$ be subharmonic in $R^{n}$ and assume $\sup u(x)=$ $+\infty$. Then there is polygonal path $\gamma$ to $\infty$ so that

$$
\lim _{\gamma} u(x)=\infty \text {. }
$$

The theorem is a generalization of Iversen's theorem. It was inspired by a recent manuscript of $B$. Fuglede where, among other results, the theorem was proved for a continuous path. Fuglede used finely harmonic functions and probability and the above result was obtained in an effort to find a classical proof. See also the work of M. N. M. Talpur (W. K. Hayman: Einige Verallgemeinerungen des Iversenschen Satzes auf subharmonische Funktionen. - Jber. Deutsch. Math.-Verein. 71, 1969, 115-122).

2. We first assume $u(x)$ continuous. Let $O_{n}$ be the open set where $u(x)>n$. There are two cases.

a) $O_{n}$ has only one component for every $n$. We then choose $x_{n}$ with $u\left(x_{n}\right)=n$ and connect $x_{n}$ to $x_{n+1}$ inside $O_{n-1}$ with a polygon. This gives the desired path.

b) Some $O_{n}$ has two components (or more). Let $A$ and $B$ be two components. By the maximum principle both are unbounded. We say that $A$ has the Phragmén-Lindelöf property if every harmonic function in $A$ which is bounded and $\leqq 0$ on $\partial A$ is $\leqq 0$. The following criterion is easy to prove.

$\mathrm{L}$ e $\mathrm{mma}$. A has the Phragmén-Lindelöf property iff the complement of $A^{-1}=\left\{\left.x|x| x\right|^{-2} \in A\right\}$ is thin at $x=0$.

Corollary. At least one of $A$ and $B$ has the Phragmén-Lindelöf property.

This follows e.g. from the Wiener criterion since

$$
\left(R^{n} \backslash A^{-1}\right) \cup\left(R^{n} \backslash B^{-1}\right)=R^{n} \backslash\{0\} .
$$


To complete the proof choose $A=A_{n}$ as above. Then $u(x)$ has to be unbounded in $A$. Choose $x_{n+1} \in A_{n}$ and let $A_{n+1}$ be the corresponding component of $O_{n+1} \cdot A_{n+1}$ also has the Phragmén-Lindelöf property and we can choose $x_{n+2} \in A_{n+1}$ etc. - The proof in this case is complete.

3. In the general case we have to find a method of constructing $\gamma$ inside the set where the potential representing $u(x)$ converges uniformly. We do this by approximating $u(x)$ by smooth subharmonic functions which are negative on the set where $u(x)$ misbehaves. The construction is quite explicit and depends on a dyadic subdivision which we are next going to describe.

We may assume that $u(x) \geqq 0$. Let $K_{v}$ be the symmetric cube of side $2^{v+1}$ and centre at $x=0$ and set $R_{v}=K_{v+1} \backslash K_{v}$. We write $(n \geqq 3)$

$$
u(x)=H_{v}(x)-\int_{K_{v+1}} \frac{d \mu(y)}{|x-y|^{n-2}}, \quad x \in K_{v+1},
$$

where $H_{v}(x)$ is harmonic in $K_{v+1}$. Set

$$
M_{v}=\operatorname{Max}_{K_{v}} H_{v}(x)+\mu\left(K_{v+1}\right) .
$$

$C$ will denote constants only depending on the dimension $n$.

We are now going to describe a subdivision of $R^{n}$ into a grid $G$ of dyadic cubes of sizes tending to zero at $\infty$. The construction depends on a given sequence of numbers $\delta_{\nu} \searrow 0$ and the sides $s(Q)$ of a cube $Q \subset K_{\nu}$ will be $<\delta_{\nu}$.

We may assume that $\delta_{v}=2^{-N_{v}}, N_{v}$ integers. For $Q \subset K_{1}$ choose $G$ so that $s(Q)=2^{-N_{1}}$. Assume that $G$ is constructed in $K_{v}$. We choose

$$
K_{v} \subset K_{v}^{(1)} \subset K_{v}^{(2)} \subset \ldots \subset K_{v}^{\left(N_{v+1}-N_{v}\right)} \subset K_{v+1}
$$

so that the cube $K_{v}^{(i)}$ has side $2^{v+1}\left(1+1 / 2+1 / 4+\ldots+1 / 2^{i}\right)$. In $K_{v}^{(i+1)} \backslash K_{v}^{(i)}$ we construct $Q \in G$ with sides $2^{-N_{\nu}-i}$ where we set $K_{v}^{\left(N_{v+1}-N_{v}+1\right)}=K_{v+1}$. This defines $G$ completely. It is important that $s(Q)$ changes slowly in the following sense. If $Q \in G, Q \subset R_{\nu}$, then $s\left(Q^{\prime}\right) \leqq 2 s(Q)$ for all $Q^{\prime} \in G$ with distance $<2^{N_{y}} s(Q)$ from $Q$.

In the formula (3.1) we now replace the measure $\mu$ by the following continuous measure $\mu^{\prime}$ :

$$
d \mu^{\prime}=\frac{\mu(Q)}{m(Q)} d x, \quad x \in Q \in G .
$$

More precisely, we define 


$$
u^{\prime}(x)=u(x)+\int_{R^{n}} \frac{d \mu(y)-d \mu^{\prime}(y)}{|x-y|^{n-2}} ;
$$

$u^{\prime}(x)$ is continuous and subharmonic if the integral converges in a suitable sense. We have for $x \in K_{\text {, }}$

$$
u^{\prime}(x)=H_{v}(x)-\int_{K_{v+1}} \frac{d \mu^{\prime}(y)}{|x-y|^{n-2}}+\int_{R^{n} \backslash K_{y+1}} \frac{d\left(\mu-\mu^{\prime}\right)(y)}{|x-y|^{n-2}} .
$$

The last term can be estimated (in $K_{v}$ ) by

$$
\left|\int_{R^{n} \backslash K_{y+1}} \frac{d \mu-d \mu^{\prime}}{|x-y|^{n-2}}\right| \leqq C \sum_{v}^{\infty} M_{i} \delta_{i}
$$

We can also show that $u(x)-u^{\prime}(x)$ is small in general. Let $x \in K_{y}$ and let $Q^{*}$ be the union of all $Q^{\prime}$ s in $G$ with distance $<M_{v} \delta$, from $x$. We find

$$
\begin{aligned}
& \left|u(x)-u^{\prime}(x)\right| \\
& \leqq \int_{Q^{*}} \frac{d \mu(y)+d \mu^{\prime}(y)}{|x-y|^{n-2}}+C \int_{K_{v+1} \backslash Q^{*}} \frac{\delta_{v}}{|x-y|^{n-1}} d \mu(y)+\sum O\left(M_{j} \delta_{j}\right) \\
& \leqq \int_{Q^{*}} \frac{d \mu+d \mu^{\prime}}{|x-y|^{n-2}}+\frac{C}{M_{v}} \int_{K_{y+1}} \frac{d \mu(y)}{|x-y|^{n-2}}+\sum O\left(M_{j} \delta_{j}\right) \\
& \leqq \int_{Q^{*}} \frac{d \mu(y)+d \mu^{\prime}(y)}{|x-y|^{n-2}}+O(1)
\end{aligned}
$$

if we assume $\sum M_{j} \delta_{j}<\infty$ and observe the definition of $M_{v}$ and $u(x) \geqq 0$.

If now $u\left(x_{v}\right) \rightarrow \infty$ it follows that if we choose $\delta_{v}$ small enough $u^{\prime}(x)$ is a subharmonic continuous function so that $u^{\prime}(x)$ is unbounded. Hence $\gamma$ exists for $u^{\prime}(x)$ and if we could make the estimate (3.4) uniformly by controling $\int_{Q^{*}}$ we would have solved our problem. This however is not possible, so an additional construction is needed to make $\gamma$ avoid these bad cubes.

4. We fix some grid $G$ and consider the set of cubes $Q \in G$ in $R_{v}$. We increase each such $Q$ in the scale $M_{v}$ and denote the resulting cubes $Q^{*}$. They cover $R_{v} M_{v}^{n}$ times $\left(\delta_{v}<M_{v}^{-1}\right)$. Denote by $\Delta_{v}^{*}$ the set of such cubes such that 


$$
\Delta_{\nu}^{*}: \quad \mu\left(Q^{*}\right) \geqq s(Q)^{n-2},
$$

and set

$$
E_{\nu}=\underset{Q^{*} \in \Delta^{*}{ }_{\nu}}{U} Q^{*}
$$

By Egorov's theorem

$$
\int_{|x-y|<M_{\nu} \delta_{y}} \frac{d \mu(y)}{|x-y|^{n-2}} \leqq M_{\nu}^{-2 n}
$$

except for $x \in E_{v}^{\prime}$ in $R_{v}$ such that $\mu\left(E_{v}^{\prime}\right) \leqq M_{v}^{-n}$ provided $\delta_{v}$ is small enough. Clearly $E_{v} \subset E_{\nu}^{\prime}$.

We can now finally fix our grid $G$ so that all conditions above are satisfied. We replace $u^{\prime}(x)$ considered above by

$$
U(x)=u^{\prime}(x)-\sum_{1}^{\infty} M_{\nu}^{n-1} \int_{E_{\nu}} \frac{d \mu^{\prime}(y)}{|x-y|^{n-2}}=u^{\prime}-p^{\prime} .
$$

Since $p^{\prime}(x)$ is the potential of a bounded measure $\left(\sum 1 / M_{v}\right.$ is supposed finite) and $u^{\prime}(x)$ is an unbounded subharmonic function, it follows easily that $U(x)$ is also unbounded. Hence there is a continuous path $\gamma$ so that $U(x) \rightarrow \infty$ along $\gamma$. The important improvement is that if $\mu\left(Q^{*}\right)>s(Q)^{n-2}$ then $\gamma \cap Q=\varnothing$. This is clear since $u^{\prime}(x) \leqq M_{\nu}$ and for $x \in Q \quad p^{\prime}(x)>$ $M_{\text {, so }} U(x) \leqq 0$.

5. It is clear that $u^{\prime}(x) \rightarrow \infty$ along $\gamma$. However $u(x)$ may not but we do have the estimate (3.4).

Let $Q_{1} \in G$ be the "first" cube intersected by $\gamma$ and let $x_{2}$ be the last point on $\gamma$ in $Q_{1}$. Then $x_{2} \in Q_{2}$ also. Let $x_{3}$ be the last point in $Q_{2}$ etc. $x_{2}$ belongs to the face $F_{1}$ of $Q_{1}$ and $F_{2}^{\prime}$ of $Q_{2} \cdot x_{3} \in F_{2}$ in $Q_{2}$ and $F_{3}^{\prime}$ in $Q_{3}$ etc. Observe that $F_{i}^{\prime \prime}=F_{i} \cap F_{i+1}^{\prime}=F_{i}$ or $F_{i+1}^{\prime}$ and that each face includes at least $100 \cdot 2^{-n} \%$ of the other.

We now join $F_{i}^{\prime \prime}$ to $F_{i+1}^{\prime \prime}$ by a line-segment $l_{i}$ in $Q_{i}$. Since $\mu\left(Q_{i}^{*}\right) \leqq$ $s\left(Q_{i}\right)^{n-2}$ it is a well-known property of Newtonian potentials that except for a small fraction of endpoints in $F_{i}^{\prime \prime}$ and $F_{i+1}^{\prime \prime}$ (with respect to normalized (n-1)-dimensional measure)

$$
\int_{Q^{*}} \frac{d \mu(y)}{|x-y|^{n-2}} \leqq C
$$

along $l_{i}$.

We now modify $x_{i}$ in the following way. Consider faces $F_{i}^{\prime \prime}$ with even index $i$. By Fubini, for every $\xi_{i} \in F_{i}^{\prime \prime}$ except a set of small relative $(n-1)$-dimensional measure there is an $l_{i}$ going forward to every $\xi_{i+1}$ 
except a small exceptional set and one $l_{i-1}$ going backward to a corresponding set of $\xi_{i-1}$ 's. We choose these $\xi_{2 i}$ 's in this manner. Then clearly they can be joined via $\xi_{2 i \pm 1}$ to each other. This now gives the desired polygon.

Institut Mittag-Leffler

Auravägen 17

S-182 62 Djursholm 1

Sweden

Received 28 August 1975 